\title{
Stepping Responses of Young and Old Adults to Postural Disturbances: Kinematics
}

\author{
Carl W. Luchies, ${ }^{*}$ Neil B. Alexander, $\dagger$ Albert B. Schultz, ${ }^{*}$ and James Ashton-Miller*
}

Objectives: When large disturbances of upright stance occur, balance must usually be restored by taking a step. We undertook this study to examine the biomechanics of stepping responses to sudden backward pulls at the waist. Primarily, response differences between young and old healthy adults were sought.

Design: A controlled laboratory study.

Subjects: Two groups of healthy and physically-fit adult females, 12 of mean age 22 (Young) and 12 of mean age 73 years (Old).

Measurements: Response kinematics were measured. From them, the stepping strategies of the subjects were derived, including the timing, length, and height of the first step taken and the rotations of major body segments and at major body joints that occurred.

Results: In response to sufficiently large backward pull forces, all subjects responded by taking one or more steps backwards. No significant age group difference appeared in the smallest disturbance for which subjects sometimes used a step response. A significant age group difference appeared in the smallest disturbance at which subjects began consist- ently to use step responses, and that disturbance was larger for the Old than for the Young. Distinct age group differences were found in stepping strategy. At large disturbance levels, the Young mostly responded by taking a single step, whereas the Old mostly responded by taking multiple steps. The steps taken by the Old, compared with those of the Young, were significantly shorter, had significantly smaller heights, and were taken significantly earlier in the responses. Body segment and joint rotations were generally modest, and few significant age group differences were found in these kinematics.

Conclusions: In restoration of perturbed balance by steptaking, the responses of the healthy, physically-fit young and old adults studied here were similar in many respects, but they differed in some important features. Joint range of motion (ROM) limitations are unlikely to explain age group differences in stepping responses to postural disturbances among healthy subjects because the ROM actually used in any of the responses observed were substantially smaller than the ROM available. J Am Geriatr Soc 42:506-512, 1994
$\mathrm{O}$ ld adults frequently fall. During a 1-year prospective study of 336 community-dwelling persons 75 years of age or older, 32 percent fell at least once. Of those who fell, 24 percent sustained serious injuries, and more than 5 percent experienced fractures. ${ }^{1,2}$ Death rates from falls per 100,000 persons in 1984 were 1.5 for those younger than 65 and 147.0 for those 85 years of age or older. ${ }^{3}$ Whatever the underlying musculoskeletal or neurological mechanisms, fall responses ultimately involve biomechanical factors.

When modest disturbances of standing posture occur, balance can be restored merely by sway reactions. The biomechanics of these responses have begun to be studied in healthy young and old adults. ${ }^{4-6}$ For larger disturbances, balance can seldom be restored without taking a step. Little is known about the biomechanics of stepping responses to postural disturbances. Force plate data have been used to describe the timing of stepping responses to impending forward falls in eight subjects of unspecified age. ${ }^{7}$ Ankle muscle myoelectric responses during step taking to recover from a forward fall have also been investigated. ${ }^{8}$ A postural stress test

From the "Department of Mechanical Engineering and Applied Mechanics, University of Michigan, Ann Arbor, Michigan; and the fDivision of Geriatric Medicine, Department of Internal Medicine and Geriatric Research Education and Clinical Center (GRECC), Ann Arbor VA Medical Center, Ann Arbor, Michigan.

Dr. Luchies' present address is Hope College, Holland, Michigan.

This work was supported in part by Public Health Service Grants AG 06621, $A G$ 08808, and AG 10542; the Dana Foundation; and the Vennema Endowment.

Address correspondence to Albert B. Schultz, Department of Mechanical Engineering and Applied Mechanics, University of Michigan, Ann Arbor, MI 48109-2125
(PST) used video taping to qualitatively observe responses to backward waist pulls in a group of young adult controls and two groups of elderly nursing home residents. ${ }^{9}$ The PST procedures were used further to examine responses of healthy young adults, healthy community-dwelling elderly with no history of falling, and elderly individuals with a history of frequent falls. ${ }^{10}$ No significant differences in balance strategy were found between the young and elderly non-fallers. It was concluded that videotape observation of such responses may not be sensitive enough to detect small age-related deteriorations in the postural control system, but may serve as a clinical tool for identifying individuals with serious balance deficits.

Thus, the biomechanics of stepping responses to impending falls have not been studied comprehensively in either young or old adults. Quantification of such responses may provide insights about causes of falls and the effects of normal aging on motor control systems. The present study was undertaken to quantify the biomechanics of healthy young and old adult responses to sudden backward waist pulls. Backward pulls were chosen because they could be controlled, introduced safely, set to any magnitude desired, and, as Wolfson et $\mathrm{al}^{9}$ pointed out, are suitable for widespread testing of elderly subjects. Because of the complexity of the data collected, the study will be described in two parts. This paper describes the response kinematics (body segment movement patterns). The response dynamics (the forces and torques developed, for example) will be described elsewhere. ${ }^{11}$

This study addressed a number of questions: (1) Are there age differences in the disturbance magnitude 
provoking a step response? (2) Are the age differences in the step strategy (single vs multiple) used to regain balance once it is lost? (3) What are the kinematics, such as step liftoff time, step landing time, and the height and length of the steps used to recover balance? (4) How do these step kinematics compare between young and old adults? (5) What joint and segment rotation excursions are required in typical stepping responses to restore standing balance upon its disturbance? (6) How do the joint and segment rotation excursions compare between young and old adults? (7) How do those joint rotation excursions compare to available joint ranges of motion?

\section{METHODS}

Subjects Twenty-four adult female volunteer subjects were tested, 12 with a mean age of 22.1 years (Young group) and 12 with a mean age of 72.9 years (Old group). Young adult subjects were selected from University staff and students. Elderly adult subjects lived in the community and were recruited from social support and recreational programs sponsored by a University-affiliated geriatrics clinic. There were no significant differences between the two groups in mean subject height and weight (Table 1). All subjects reported themselves as being right foot dominant. A medical history was obtained in all subjects, and a physical examination that focused on musculoskeletal and neurological items was conducted on all elderly subjects. All subjects denied significant head trauma, otologic or neurologic disease, limb fracture, musculoskeletal impairments, or persistent problems with vertigo, lightheadedness, unsteadiness, or falling. Half of the elderly subjects admitted to visual impairment correctable with lenses such that single or binocular distance vision was found to be $20 / 70$ or better on Snellen chart testing. Up to one-third of the elderly group noted occasional osteoarthritic symptoms, one-fourth of the group had bilateral and apparently age-associated hearing loss, one-fourth had minimal bilateral proximal weakness in the lower extremities with scores no worse than $4+$ on a scale of 0 (no muscle contraction) to 5 (maximal resistance without fatigue), and approximately one-half had reduced $128-\mathrm{Hz}$ vibration sense at the toe. Despite these subtle abnormalities on history and physical examination, all elderly subjects were physically active in either regular walking or exercise programs.

Disturbances Imposed Subject stood upright, looking ahead with eyes open, arms folded across the chest, and bare feet placed in marked comfortable

TABLE 1. ANTHROPOMETRIC DATA

\begin{tabular}{lcc} 
& Young & Old \\
\hline Number $(n)$ & 12 & 12 \\
Mean age (yrs) & $22.1(2.5)$ & $72.9(4.9)$ \\
Age range (yrs) & $19-26$ & $65-80$ \\
Mean height $(\mathrm{cm})$ & $163.2(3.8)$ & $160.8(7.6)$ \\
Mean body mass $(\mathrm{kg})$ & $56.5(4.8)$ & $61.2(8.1)$ \\
\hline
\end{tabular}

Standard deviations in parentheses. There were no significant age group differences in subject height or body mass. positions. They wore a suspended harness designed to safely arrest any major fall but not to interfere with the responses under observation. They wore a second harness around the waist incorporating anterior padded ( $6 \mathrm{~mm}$ of neoprene) blocks adjusted to provide secure contact with their anterior-superior iliac spines for transmission of the disturbing force to the body (Figure 1).

The timing of stimulus presentation was standardized by coordinating it with body sway. A string potentiometer attached to the waist harness sensed the anterior/posterior movement of the waist. Subjects stood relaxed for 1 minute while waist movement during normal sway was measured. Each backward pull was then applied to the waist harness the instant the subject swayed forward through her mean sway position with a waist-level velocity less than $0.01 \mathrm{~m} / \mathrm{s}$. A load cell attached to the harness measured the time history of this backward pull.

Backward pulls were produced by a computer-released dropped weight and cable system designed to allow the subject to sway freely prior to the release of the weight. The weight dropped was always 20 percent of the subject's body weight. It began to pull the subject backwards after dropping $1 \mathrm{~cm}$, which together with the release mechanism time delay took approximately $80 \mathrm{~ms}$, and then continued to drop. The height of the subsequent drop was set in relation to subject height to correspond to various whole-body backward sways about the ankles (equivalent disturbance angle, or

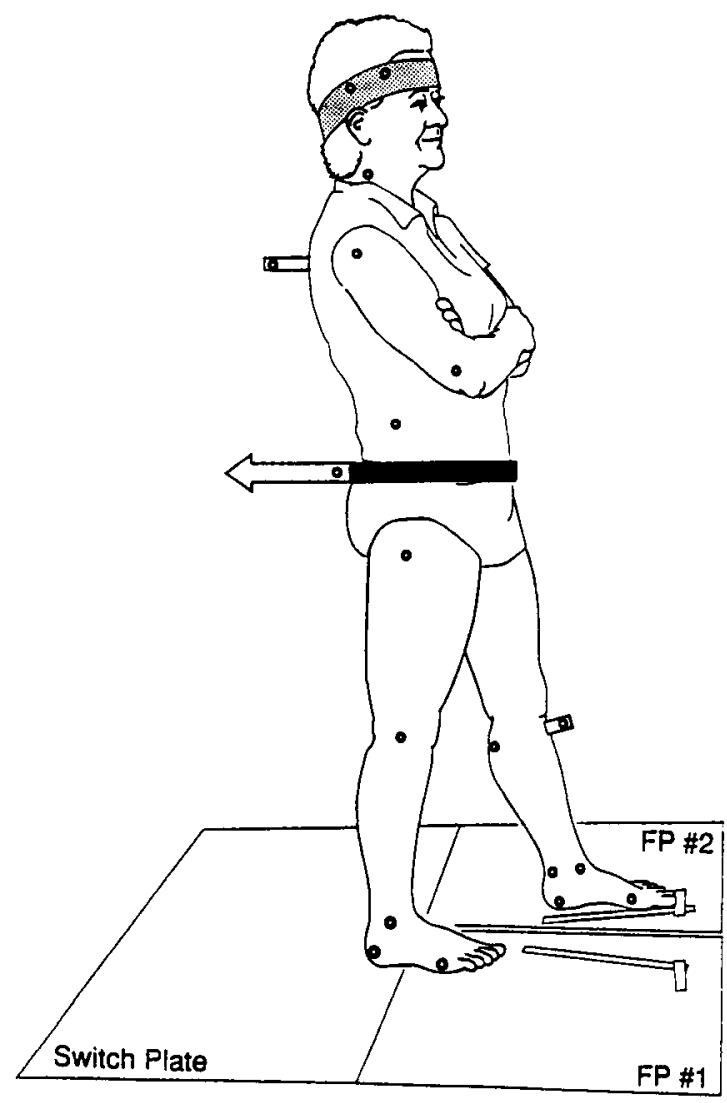

FIGURE 1. Schematic showing LED locations, waist harness, and applied force. 
EDA). The EDA used, in the fixed, initially randomized order in which they were imposed, were $3.0,3.5,0.25$, $1.5,1.0,1.25,0.5,2.5,2.0$, and 0.75 degrees, corresponding to mean pull distances ranging from 0.4 to $6.0 \mathrm{~cm}$. At each EDA, three consecutive trials were conducted. Several practice trials at different EDA were first run to acquaint the subject with the test procedures. All data acquisition units were triggered at the same time as the drop mechanism.

Kinematic Measurements Body segment kinematics were measured using a four-camera Watsmart (Northern Digital) optoelectronic motion analysis system that tracked the locations in three dimensions of 20 infrared-emitting diodes (IREDs). This system, when used in studies of the present type, can measure displacements to within $2 \mathrm{~mm}$ and body segment rotations to within $0.7 \mathrm{deg}$. IREDs were placed on each leg near the heel, ball of the foot, ankle, and knee and on the right hip. Because the cameras viewed primarily from the right side, two ankle and knee IREDs were placed on the medial left leg so that at least one would be visible when a step was taken. IREDs were placed on the right side of the trunk, on a wand attached to the back at the T5 level, on the right shoulder and elbow, and on the right side of the neck. Two IREDs were placed on the head, one on the waist harness, and one on the force plate (Figure 1).

The reference orientation of each body segment was established from the mean position of the segment IREDs during the first $50 \mathrm{~ms}$ after initiation of a trial, before the onset of the pull force. The rotation changes that occurred after this time were then calculated. Segment rotations counterclockwise when viewed from the right were considered to be positive. Joint relative rotation changes were calculated using the absolute rotation data for the segments proximal and distal to each major body joint. Joint rotations counterclockwise from a superiorly-directed longitudinal line through the distal segment to one through the proximal segment were considered to be positive. In other words, ankle plantarflexion, knee flexion, and hip, shoulder, and neck extension were positive joint rotations.

Other Measurements Made Measurements were also made of left and right foot floor reactions, using two AMTI six-component force plates, and of steppedfoot floor recontact time using a switch plate. Those measurements will be described elsewhere. ${ }^{12}$ However, the step timing data presented in this paper were derived form the floor reaction and recontact measurements. Myoelectric data were also obtained but will not be reported here. Steps were considered to be initiated when the vertical floor force on the stepped foot decreased to $30 \mathrm{~N}$. All responses were also videotaped. The videotape recording was used to determine which foot was used for the initial step of any step responses and how many additional steps the subject used to regain her balance.

Data Analysis Data from all three trials at each EDA were used to analyze the number and type of steps taken. All other reported quantities were derived from data for only the second of the three consecutive trials at each EDA. All load cell, force plate, and IRED data were sampled at $200 \mathrm{~Hz}$ for 3 seconds following the trigger signal. The IRED data were processed to calculate time histories of the body segment absolute rotations and the joint relative rotations that occurred. When steps were taken, step distance and height data were derived from the ankle IRED location data.

All raw data were filtered digitally using a second order low pass Butterworth filter $(4-\mathrm{Hz}$ cutoff frequency) with forward and backward reflection to minimize initial and final-time artifacts. Phase shift was minimized by using forward and backward passes. The segment absolute orientation angles were also filtered after they were calculated, based on each link's proximal and distal locations. All times were measured from the onset of the disturbance. Time histories were scanned until the recontact time of the first step to find peak positive, peak negative, and total excursion values of the kinematic parameters. All first steps were completed within $600 \mathrm{~ms}$ after triggering. Values of the joint and segment angles at the instant of liftoff for the first step were also noted.

Parameter means and standard deviations were calculated for each age group. Analysis of Variance (ANOVA) was conducted to examine the effect of age and balance strategy (no step, single step, or multiple steps) on the disturbance descriptors: duration, peak force, and impulse strength (area under the force-time curve) over all 10 disturbance levels. ANOVA was used to examine the effect of age and stepping strategy (single or multiple steps) on the following step kinematics: liftoff time, landing time, duration, length, and height in response to the four largest disturbance levels. Since the step kinematics did not vary with different step strategies, repeated measures ANOVA across all strategies was used to examine, at the four largest disturbance levels, the effect of age on these step kinematics. Using ANOVA, mean age group differences in body segment and joint rotations excursions and the body segment and joint rotations at the time of step liftoff were assessed for the largest disturbance while controlling for differences in step strategy. Two-tail $P$ values $<0.05$ were considered statistically significant throughout. A non-parametric Mann-Whitney U Test was used to determine age differences in the disturbance level at which step taking began (step threshold). A Fisher's Exact Test was used to assess age differences in the percent of subjects' use of single and multiple step responses.

\section{RESULTS}

Disturbance Data At each EDA, all subjects experienced essentially the same disturbance independent of their age or their resulting balance strategy. ANOVA was used to determine the relationship of the applied force duration, peak force magnitude, and the impulse strength to the disturbance level (EDA), the subject group (Young-Old), and the resulting balance strategy (sway, single step, or multiple step) (Table 2). The force duration, peak magnitude, and impulse strength were independent of age and balance strategy but were strongly related to the disturbance level (for example, $r=0.92$ for correlation of impulse strength 
TABLE 2. MEAN CHARACTERISTICS OF THE DISTURBANCES

\begin{tabular}{|c|c|c|c|c|}
\hline & \multicolumn{4}{|c|}{ Equivalent Disturbance Angle (deg) } \\
\hline & 2.0 & 2.5 & 3.0 & 3.5 \\
\hline \multicolumn{5}{|c|}{ Force duration (ms)* } \\
\hline Young & $138(17)$ & $168(9)$ & $189(14)$ & $198(22)$ \\
\hline Old & $142(13)$ & $167(14)$ & $170(20)$ & $193(16)$ \\
\hline \multicolumn{5}{|c|}{ Peak force $(N)^{*}$} \\
\hline Young & $160(14)$ & $154(15)$ & $149(13)$ & $156(26)$ \\
\hline Old & $162(18)$ & $160(20)$ & $170(19)$ & $165(19)$ \\
\hline \multicolumn{5}{|c|}{ Impulse strength (Ns)* } \\
\hline Young & $11.9(1.4)$ & $13.4(1.6)$ & $14.5(1.6)$ & $15.5(1.7)$ \\
\hline Old & $13.1(2.3)$ & $14.8(2.8)$ & $15.6(2.7)$ & $17.1(2.8)$ \\
\hline
\end{tabular}

Standard deviations in parentheses.

Based on ANOVA: * No age or response strategy effect.

to disturbance level). The mean time after triggering at which the backward pull force began ranged from 73 to $99 \mathrm{~ms}$ in the two groups.

Step Threshold No significant age group differences were apparent in the smallest disturbance level at which subjects used a step response in any of the three trials to regain their balance (median EDA: 0.88 for Young, 1.00 for Old, $P>0.1$ ). The disturbance magnitude provoking a step response for all three trials and for all trials with a disturbance larger than that one was significantly different between the two groups, although the difference was small (median EDA: 1.25 for Young, 1.50 for Old, $P<0.05$ ).

Step Taking The three largest EDA uniformly provoked step-taking, with one exception, by all Young and all Old subjects (data available from authors). Some subjects, both Young and Old, stepped even at the smallest EDA. Other subjects, both Young and Old, did not step until the EDA reached $2.5 \mathrm{deg}$.

Whenever they took steps to regain balance, the Young usually took a single step (Figure 2). The Old switched from a predominantly single-step response at $\mathrm{EDA} \leq 2.0$ to a multiple-step response at EDA $\geq 2.5$ to regain balance at the larger disturbance levels (Figure 3). In fact, for the three largest EDA (EDA $\geq 2.5)$, 83 percent of the Old took multiple steps compared with 33 percent of the Young $(P<0.05)$.

In the remaining subsections of Results, step kinematic parameter data will be presented for EDA values

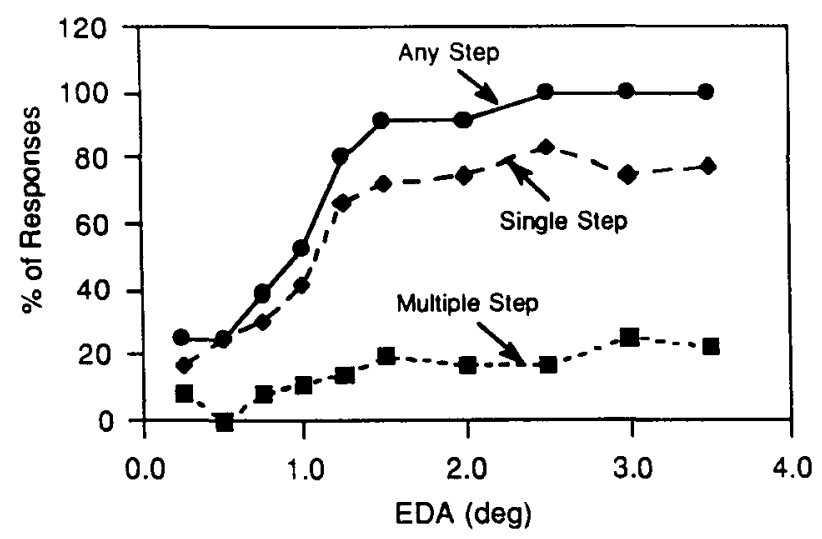

FIGURE 2. Stepping strategies in the Young.

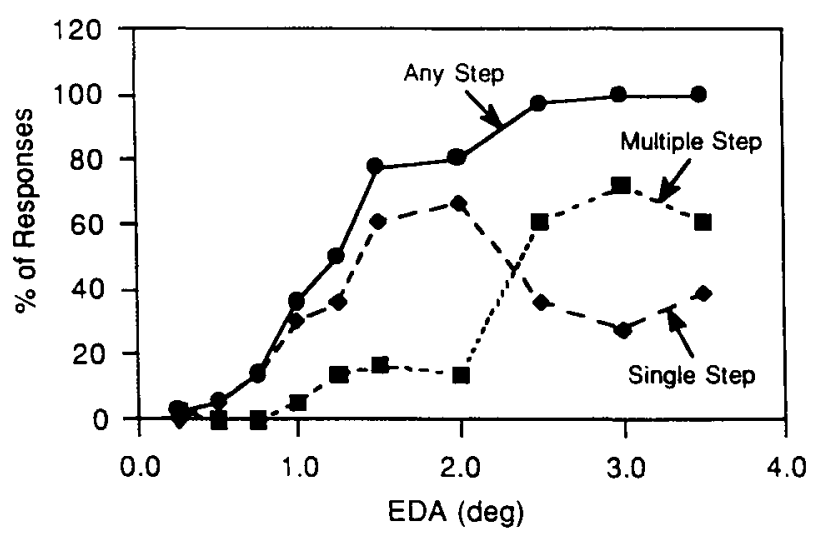

FIGURE 3. Stepping strategies in the Old.

at 2.0, 2.5,3.0, and $3.5 \mathrm{deg}$. Segment and joint rotation data will be presented for EDA $=3.5$.

First-Step Liftoff Time Significant age effects were found in the first-step liftoff time, with the Old stepping earlier than the Young. Over the four largest disturbance levels considered, the liftoff time ranged from 358 to $405 \mathrm{~ms}$ in the Young and from 289 to 370 $\mathrm{ms}$ in the Old (Table 3). For EDA of 3.0 and $3.5 \mathrm{deg}$, the Old in the mean stepped significantly earlier than the Young, by 23 percent $(95 \mathrm{~ms}$ ) and 19 percent $(69$ $\mathrm{ms})$, respectively.

First Step Duration and Landing Time Significant age effects were found in the first step landing time at all EDA levels and in step duration time at EDA of 2.5 and 3.0, with the Old landing earlier and having a shorter step duration than the Young. The landing time ranged from 523 to $568 \mathrm{~ms}$ in the Young and from 440 to $492 \mathrm{~ms}$ in the Old. The step duration ranged from 163 to $175 \mathrm{~ms}$ in the Young and from 122 to 150 $\mathrm{ms}$ in the Old (Table 3).

Step Height and Length Significant age effects were found in the first-step length and height at essentially all EDA levels, with the Old step height smaller and the step length shorter then the Young. In the mean, the step height ranged from 3.9 to $5.3 \mathrm{~cm}$ for the Young and from 1.7 to $2.7 \mathrm{~cm}$ for the Old. Step length ranged from 22.9 to $29.3 \mathrm{~cm}$ for the Young and from 13.9 to $16.3 \mathrm{~cm}$ for the Old (Table 3). 
TABLE 3. MEAN STEP KINEMATIC PARAMETERS

\begin{tabular}{|c|c|c|c|c|}
\hline & \multicolumn{4}{|c|}{ Equivalent Disturbance Angle (deg) } \\
\hline & 2.0 & 2.5 & 3.0 & 3.5 \\
\hline \multicolumn{5}{|c|}{ Liftoff time (ms) } \\
\hline Young & $382(75)$ & $370(68)$ & $* * * 405(85)$ & $* * * 358(65)$ \\
\hline Old & $317(87)$ & $370(171)$ & $310(120)$ & $289(86)$ \\
\hline \multicolumn{5}{|c|}{ Landing time (ms) } \\
\hline Young & *558 (101) & $* * 539(47)$ & $* * * 568(87)$ & $* * * 523(67)$ \\
\hline Old & $464(97)$ & $492(207)$ & $448(118)$ & $440(95)$ \\
\hline \multicolumn{5}{|c|}{ Step duration (ms) } \\
\hline Young & $175(51)$ & **169 (53) & *163 (34) & $166(28)$ \\
\hline Old & $147(22)$ & $122(50)$ & $138(28)$ & $150(32)$ \\
\hline \multicolumn{5}{|c|}{ Step height $(\mathrm{cm})$} \\
\hline Young & $3.9(2.2)$ & $* * * 4.3(1.5)$ & $* * 5.1(2.7)$ & $* * 5.3(2.1)$ \\
\hline Old & $2.6(1.6)$ & $1.7(1.1)$ & $2.4(1.1)$ & $2.7(1.0)$ \\
\hline \multicolumn{5}{|c|}{ Step length (cm) } \\
\hline Young & ${ }^{*} 22.9(9.8)$ & **25.1 (9.2) & $* * * 26.9(6.6)$ & $* * * 29.3(7.8)$ \\
\hline Old & $13.9(6.2)$ & $15.8(7.1)$ & $16.3(5.7)$ & $16.2(5.9)$ \\
\hline
\end{tabular}

Standard deviations in parentheses.

Based on Repeated Measures ANOVA, Young $>$ Old: ${ }^{*} P<0.05,{ }^{* *} P<0.01,{ }^{* * *} P<0.001$.

Segment Rotations at Step Initiation At the instant of liftoff for the first step, body segment rotations from disturbance onset reference values were, in the mean and in both age groups, less than $20 \mathrm{deg}$, with few significant age group differences (Table 4 provides data for the 3.5 deg EDA).

Segment Rotation Excursions Mean rotation excursions, defined as the sum of the maximum positive and maximum negative rotations, were less than 32 deg for all body segments. The segment rotation excursions in the Young tended to be larger than those in the Old for all segments except the arms (Table 4). Statistically significant age effects occurred in the stepped lower leg (32 and $17 \mathrm{deg}$ in Young and Old), the stepped upper leg (20 and $15 \mathrm{deg}$ ) and the stance foot (15 and $7 \mathrm{deg}$ ).

Joint Rotations at Step Initiation The mean relative joint rotations that had occurred at the time the first step was initiated were generally less than $14 \mathrm{deg}$ in both age groups, except at the hips, which were flexed as much as $27 \mathrm{deg}$ (Table 5). The Young tended to have larger joint rotations at step initiation than the Old, with differences being significant at the stepped knee and hip and the stance hip.

Joint Rotation Excursions Stance leg joint rotation mean excursions were less than $15 \mathrm{deg}$ at the ankle and knee and less than $29 \mathrm{deg}$ at the hip (Table 5). Stepped leg joint rotation mean excursions ranged to $11 \mathrm{deg}$ at the ankle, $33 \mathrm{deg}$ at the knee, and $30 \mathrm{deg}$ at the hip. Shoulder and neck mean extensions were 10 deg or less. The Young tended to have larger ankle, knee, and hip rotations in both legs than the Old, but the differences were seldom significant.

\section{DISCUSSION}

No previous quantitative comparisons of stepping responses to large postural disturbances between healthy young and elderly appear to have been reported in the literature. Our kinematic analyses compared the step threshold, step strategy, body segment
TABLE 4. MEAN SEGMENT ROTATIONS AT STEP INITIATION AND EXCURSIONS (DEG) AT AN EQUIVALENT DISTURBANCE ANGLE OF 3.5 DEG

\begin{tabular}{|c|c|c|}
\hline & At Step Initiation & Excursions \\
\hline \multicolumn{3}{|l|}{ Step foot } \\
\hline Young & $2.1(6.1)$ & $24.1(13.5)$ \\
\hline Old & $0.8(4.6)$ & $16.2(8.9)$ \\
\hline \multicolumn{3}{|c|}{ Step lower leg } \\
\hline Young & $2.0(4.2)$ & *32.0(17.4) \\
\hline Old & $4.5(3.7)$ & $17.0(5.2)$ \\
\hline \multicolumn{3}{|c|}{ Step upper leg } \\
\hline Young & ***15.1(3.9) & $* 20.5(8.7)$ \\
\hline Old & $19.9(4.4)$ & $15.2(2.8)$ \\
\hline \multicolumn{3}{|c|}{ Stance foot } \\
\hline Young & $4.0(5.4)$ & ${ }^{*} 15.1(9.3)$ \\
\hline Old & $3.1(5.4)$ & $7.3(5.1)$ \\
\hline \multicolumn{3}{|c|}{ Stance lower leg } \\
\hline Young & $5.6(4.6)$ & $10.4(6.2)$ \\
\hline Old & $5.3(2.7)$ & $8.2(2.6)$ \\
\hline \multicolumn{3}{|c|}{ Stance upper leg } \\
\hline Young & **13.7 (2.4) & $17.6(2.9)$ \\
\hline Old & $9.3(3.4)$ & $14.7(3.7)$ \\
\hline \multicolumn{3}{|l|}{ Trunk } \\
\hline Young & $-11.9(3.5)$ & $12.5(3.6)$ \\
\hline Old & $-9.5(2.3)$ & $11.7(2.9)$ \\
\hline \multicolumn{3}{|l|}{ Arms } \\
\hline Young & $-12.3(3.8)$ & $13.2(4.2)$ \\
\hline Old & $-7.7(8.0)$ & $14.2(6.8)$ \\
\hline \multicolumn{3}{|l|}{ Head } \\
\hline Young & $*-5.8(2.9)$ & $10.0(6.1)$ \\
\hline Old & $-3.1(3.8)$ & $7.8(4.6)$ \\
\hline
\end{tabular}

Standard deviations in parentheses. Means are over all step responses. Positive rotations are counterclockwise when viewed from the right. Based on ANOVA Young $>$ Old: ${ }^{*} P<0.05,{ }^{* *} P<0.01$. Young $<$ Old: ${ }^{* * *} P<0.05$

and joint rotation angles, and step height, length, and timing in these two groups.

The salient age-group difference in the response kinematics examined was the difference in stepping strategy for the larger disturbances, with the Old taking mostly multiple steps and the Young taking mostly 
TABLE 5. MEAN JOINT ROTATIONS AT STEP

INITIATION AND EXCURSIONS (DEG) AT AN EQUIVALENT DISTURBANCE ANGLE OF 3.5 DEG

\begin{tabular}{|c|c|c|}
\hline & At Step Initiation & Excursions \\
\hline \multicolumn{3}{|l|}{ Step ankle } \\
\hline Young & $-0.8(7.8)$ & $10.9(2.9)$ \\
\hline Old & $3.4(3.7)$ & $7.6(3.5)$ \\
\hline \multicolumn{3}{|l|}{ Step knee } \\
\hline Young & ${ }^{*} 13.2(6.9)$ & $33.0(8.3)$ \\
\hline Old & $5.7(5.7)$ & $26.0(7.2)$ \\
\hline \multicolumn{3}{|l|}{ Step hip } \\
\hline Young & $*-27.0(6.2)$ & $29.3(6.8)$ \\
\hline Old & $-19.5(5.0)$ & $26.3(4.8)$ \\
\hline \multicolumn{3}{|c|}{ Stance ankle } \\
\hline Young & $-0.8(12.7)$ & ${ }^{*} 14.4(7.1)$ \\
\hline Old & $2.2(6.7)$ & $9.1(4.0)$ \\
\hline \multicolumn{3}{|c|}{ Stance knee } \\
\hline Young & $8.1(6.0)$ & $12.8(5.0)$ \\
\hline Old & $3.9(4.0)$ & $9.1(4.8)$ \\
\hline \multicolumn{3}{|c|}{ Stance hip } \\
\hline Young & $* *-25.8(3.9)$ & $28.2(5.2)$ \\
\hline Old & $-18.9(4.7)$ & $25.6(5.7)$ \\
\hline \multicolumn{3}{|l|}{ Shoulder } \\
\hline Young & $-0.3(1.2)$ & $3.2(1.4)$ \\
\hline Old & $1.8(7.4)$ & $7.3(6.4)$ \\
\hline \multicolumn{3}{|l|}{ Neck } \\
\hline Young & $6.2(5.2)$ & $9.9(7.4)$ \\
\hline Old & $6.3(5.5)$ & $9.5(5.1)$ \\
\hline
\end{tabular}

Standard deviations in parentheses. Means are over all step responses. Positive rotations are in ankle plantarflexion, knee flexion, and hip, trunk, shoulder and head extension.

Based on ANOVA Young $>$ Old: ${ }^{*} P<0.05,{ }^{* *} P<0.01$.

single steps. Multiple step-taking has been cited, ${ }^{9}$ but not confirmed, as an indication of poor balance. Taking multiple steps does seem a more conservative strategy than taking single steps. There are more opportunities to arrest energy each time a stepped foot recontacts the ground. When multiple steps are taken, more adjustments can be made to correct for any ill-chosen early responses. Since the multiple steps can be shorter, they might require smaller body segment and joint rotations and smaller joint torques, and so decrease the biomechanical requirements of multiple compared to single step responses.

Our findings support, in some respects, and controvert in others the hypothesis that old adults choose more conservative responses in restoring balance when it is perturbed. The choice by the Old of a more conservative response (multiple steps) to disturbances that more fully stress their balance supports the hypothesis. The finding that the Old in the mean started stepping at the same threshold disturbance and began to step consistently at a somewhat larger mean disturbance level than did the Young does not support the hypothesis. Since step responses can arrest much more energy than sway responses, the more conservative strategy would be to take steps in response to even small disturbances. Finally, in the present study neither the rotation magnitude nor the joint torque ${ }^{11}$ differences among the different stepping strategies were large. Thus, whether or not the hypothesis is supported with regard to the biomechanical requirements of the different stepping strategies is not clear.
The mean step foot liftoff time was significantly earlier in the Old than the Young. In a comparable group of subjects, simple reaction times in lower extremity joints of healthy elderly ( 212 to $253 \mathrm{~ms}$ ) were longer than those of healthy young adults (196 to 219 $\mathrm{ms}$ ) by 30 to $40 \mathrm{~ms} .^{13}$ Despite their presumably longer simple reaction times, the Old in the present study stepped earlier then the Young.

While the body segment absolute rotation and the joint relative rotation excursions used in these responses were sometimes significantly different between the two age groups, the magnitudes of those differences were not large (Tables 4 and 5). The joint ranges of motion (ROM) used by both Young and Old subjects in the present study were modest compared with the joint ROM available (Figure 4). ${ }^{12}$ This makes it unlikely that limitations in joint ROM explain YoungOld differences in responses to the postural disturbance examined here.

The findings from this study support a conclusion reached in other studies of responses to postural disturbances by healthy young and healthy old adults: the size and even the existence of significant age group differences is dependent on the specific nature of the postural perturbation. Moreover, these differences seem to bear no obvious relation to reasonable presumptions as to the challenge of the task or as to what response strategies might be adopted. The discussion above regarding the different stepping strategies adopted by the two age groups illustrates this in the present study. As a further example, from previous studies, substantial age group differences in responses were found when subjects attempted to maintain balance while standing on a stationary narrow support, smaller differences were found when standing on that support while it was accelerated, and still smaller differences were found when standing flat on an accelerated surface. ${ }^{5,6}$ The first of these tasks intuitively would seem to have been less challenging than the other two.

Normalization of the observed kinematic response parameters to individual subject anthropometry did not seem warranted since the subject anthropometric

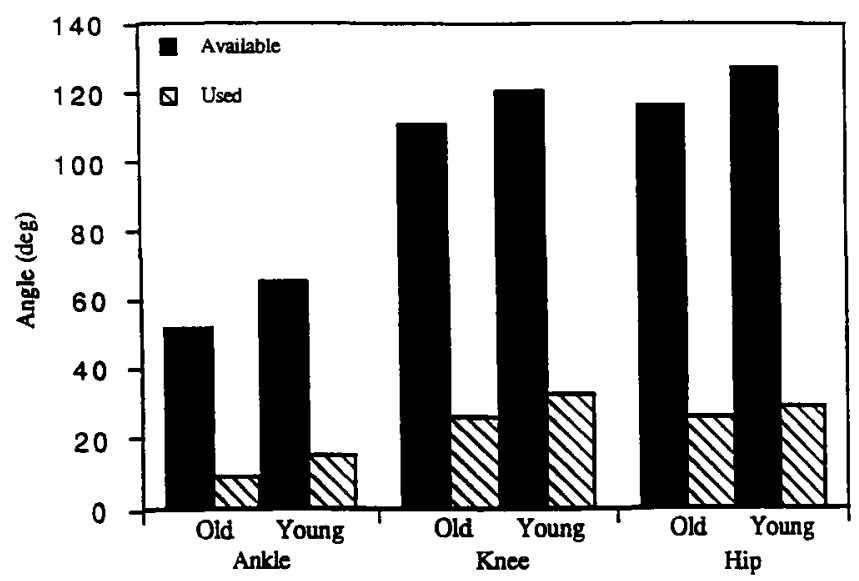

FIGURE 4. Comparison of the maximum joint ranges of motion used (Used) (either step or stance leg) with the joint range of motion available ${ }^{12}$ (Available) for Young and Old subjects. 
data were not significantly different between the two groups. Body segment and joint rotation data are inherently non-dimensional.

\section{ACKNOWLEDGMENTS}

The contributions of Linda Ford, Viviane Franca, Janet Grenier, Youda He, Keli Schneberger, David Warwick, and Sara Zeilstra in this research are gratefully acknowledged.

\section{REFERENCES}

1. Tinetti ME, Ginter SF. Identifying mobility dysfunctions in elderly patients-Standard neuromuscular examination or direct assessment? JAMA 1988;259:1190-1193.

2. Tinetti ME, Speechley M. Prevention of falls among the elderly. N Engl ] Med 1989;320:1055-1059.

3. Lambert DA, Sattin RW. Deaths from falls, 1978-1984. MMWR CDC Surveill Summ 1988;37:21-26.

4. Romick-Allen RA, Schultz AB. Biomechanics of Reactions to Impending Falls. J Biomech 1988;21:591-600.
5. Alexander NB, Shepard NT, Gu MJ, Schultz AB. Postural control in young and elderly adults when stance is perturbed: Kinematics. J Gerontol Med Sci 1991;47:M79-87

6. Gu MJ, Schultz AB, Shepard NT. Alexander NB. Postural control in young and elderly adults when stance is perturbed: Dynamics. J Biomech, 1994 manuscript accepted.

7. Do MC, Breniere $Y$, Brenguier P. A biomechanical study of balance recovery during the fall forward. J Biomech 1982;15:933-939.

8. Do MC, Breniere $\mathrm{Y}$, Bouisset $\mathrm{S}$. Compensatory reactions of forward fall: Are they initiated by stretch receptors? Electroenceph Clin Neurophys 1988:69:448 451.

9. Wolfson LI, Whipple R, Amerman P, Kleinberg A. Stressing the postural response, a quantitative method for testing balance. I Am Geriatr Soc 1986;34:845-850.

10. Chandler JM, Duncan PW, Studenski SA. Balance performance of the postural stress test: Comparison of young adults, healthy elderly, and fallers. Phys Ther 1990;70:410-415.

11. Luchies CW, Alexander NB, Schultz AB, Ashton-Miller JA. Stepping responses of young and old adults to postural disturbances: Dynamics. I Biomech, 1994, manuscript accepted.

12. Chen HC, Ashton-Miller JA, Alexander NB, Schultz AB. Stepping over obstacles; Gait patterns of healthy young and old aduits. J Gerontol Med Sci 1991;46:M196-203.

13. Ashton-Miller JA, Alexander NB, Grenier J et al. Pre-motor, motor and simple reaction times in the lower extremities of young and old adults. I Gerontol Med Sci, 1994, manuscript accepted. 